\title{
A Tentative Study of Linguistic Error Analysis and Teacher's Attitude
}

\author{
Zhao Gao ${ }^{1}$, and Chuanmao Tian $^{2}$ \\ ${ }^{1)}$ School of Foreign Studies, Yangtze University, Jingzhou, Hubei, P. R. China (531278464@qq.com) \\ ${ }^{2)}$ School of Foreign Studies, Yangtze University, Jingzhou, Hubei, P. R. China (tcm_316@163.com)
}

\begin{abstract}
On the basis of a distinction between error and mistake, the linguistic mistakes/errors made by the students together with their causes are analyzed. The attitude which the teacher should have toward the linguistic mistakes/errors made by the students are explored. It is suggested that in English teaching, especially in oral English teaching practice, the teacher is supposed to take a correct attitude toward students' linguistic mistakes/errors. The teacher can use the heuristic or guiding method to help students correct their mistakes/errors. The teacher should try their best to avoid pointing out mistakes/errors directly. In non-oral English communication, the teacher is expected to deal with students' mistakes/errors very seriously.
\end{abstract}

Keywords - linguistic error/mistake, reason, teacher, attitude

\section{小议语言错误分析与教师的态度}

\author{
高照 $^{1}$ 田传茂 ${ }^{2}$ \\ 1) 长江大学外国语学院, 荆州, 湖北, 中国 \\ 2) 长江大学外国语学院, 荆州, 湖北, 中国
}

摘 要 本文通过区分误差和失误, 分析了学生的语言错误及其原因, 探讨了教师对学生所犯语言错误应持的态度。本文认为, 在 英语教学, 特别是口语教学实践中, 教师对学生的语言错误应持正确的态度。在口语交际中可以用引导的方法来帮助学生纠正错误, 要尽量避免直截了当地指出和纠正错误。在非口语交际中, 教师应当非常严肃认真地对待和纠正学生的语言错误。

关键词 语言错误, 成因, 教师, 态度

\section{1. 引言}

无论是学习本族语还是外语, 都不可避免地会出现一 些误差。第二语言或外语学习者所使用的是一种过度性语 言, 是处于母语和目标语之间的中介语。学生在口语和书 面语中的误差往往表现在没有掌握好某一个语言项目。有 人把这些语言错误归纳为主谓不一致, 时态不一致, 词性 误用等 17 种类型（Cao 1993: 36)。下面结合成人英语教学 实践对学生口语和非口语中的错误进行归纳并顺带探讨一 下教师应当如何正确对待学生所犯的语言错误。

\section{2. 误差与失误}

人们常常把误差 (error) 和失误 (mistake) 混淆起来, 把他们都称为错误。其实, 误差和失误是两个不同的概念, 前者指由于语法结构、词汇等言语层次的知识不完全而引
起的, 学习者要经过老师指出后才知道自己的错误所在, 而后者则指由于学习者在写或读时, 由于太疲劳, 注意力 不集中, 粗心或心情紧张而造成的, 他们通常很快就能觉 察到（He and Yan 1991: 38）。

\section{3. 误差的起因及分类}

语言学家和心理学家们认为, 学习是一个不断犯错误、 不断纠正错误的过程。他们还认为不同的学习过程会造成 不同的误差, 并以此来划分和解释各类误差 (Zhang 1993: 79)。误差的起因大致包括以下几个方面。

\section{1 母语干扰性误差}

这类错误又称为语际错误, 主要指受母语（本族语或 方言）的影响而造成的错误。如汉语的 “他长得很强壮”, 
学生常会译成 “He grows very strong.”。正确的表达方式是 “He is very strong”。又如 “这间教室很明亮”, 学生可能 会译成 “The classroom very bright”, 正确的译法是 “The classroom is very bright.”。造成此种误差的原因有以下几点。

\section{1 .1 语音方面的干扰}

不少中国学生会觉得 $[\theta]$ 和 $[ð]$ 这两个音素的发音很费 力, 容易把 three 发成[tri:], 把 this 读成[dis], 其原因可能 是汉语里没有这两个音素而造成的失误。方言发音对英语 学习也有影响。例如湖北学生经常将鼻音[n]读成舌边音 [1]。 操客家方言的学生常把[ou]读成[o:], 因为客家话里没有 [ou] 这个音素。还有学生 he/she 不分, 因为汉语中 “他” / “她” 发音相同。

\section{1 .2 时态方面的干扰}

汉语语法里没有时态这个范畴。表示过去, 可用时间 状语 “昨天”, “上个月”, “去年” 等表示。因此, 中国学 生往往在时态上出错, 如把 “他昨天去看了电影” 写成 “ $\mathrm{He}$ go to the cinema yesterday.”。

\subsection{3 表达方式方面的干扰}

由于受汉语思维方式的影响, 学生在学习英语的过程 中犯中式英语 (Chinglish) 的错误, 如 “你先走” (You go first.), 学生们稍不留神就会写成 “You first go.”。又如汉 语的 “名词+形容词” 句子结构中, 名词和形容词之间是不 用系动词的, 因此学生常说 “The classroom very bright” 这 样的病句。

\section{2 语内错误}

这类错误主要指学习者对目标语掌握不全面或一知半 解而造成的错误, 如学生对 like 的词性掌握不全面, 就有 可能把 “He likes the dog” 说成 “He is like the dog.”, 从而 闹出笑话。有时, 学生凭借自己所掌握的目标语的知识, 进行过度概括, 也会造成此类错误。例如学生根据学过的 语言结构依样画漂, 结果推导出错误的结构:

误: Will you explain me the answer?

正: Will you explain the answer to me?

学生之所以会犯这样的错误, 是因为他们学过这种结 构:

We gave the girl the book.

We gave the book to the girl.

还有的错误是由于教师的误导而造成的, 如过分操练 进行时, 学生可能会造出这样的句子: I am knowing him. 或 I am liking him.。

\section{4. 教师的态度}

教师应该如何对待学生的错误? 实践表明, 纠错会使 学生的学习热情减退, 影响他们的学习兴趣, 挫伤他们的 自尊心, 甚至会给他们带来精神压力 (Dai 1990: 4)。尽管 如此, 大多数学生还是希望并且要求老师帮助他们改正错 误, 因此, 我们认为, 教师在教学过程中对学生所犯的错 误应持正确的态度。从交际的角度看, 口语中所犯的错误 如果不是语用失误, 如: Foreigner: You are pretty. / Student: No, no, not all., 那么在不影响交际的情况下可以不纠正学 生的错误, 更不应该担心学生的错误, 因为口语是为了交 流思想, 能进行思想交流, 交际就成功了。不纠正学生的 错误, 反而能更好地培养学生用语言交流的能力。如果教 师在交际过程中不断纠正错误, 学生可能会怀疑自己的语 言交际能力, 甚至失去信心。

当然, 教师可以在适当的时候运用学生觉察不到的不 影响交际的方法来纠正学生的语言错误, 如: Student: Who first go? / Teacher: Li Ming goes first, then Zhang Ling.。在这 个交际过程中, 学生犯了语序错误。在汉语里, 我们说 “谁 先去”, 但英语里应该说 “谁去先” (Who goes first?), 而且 学生的说话有时态错误, go 应改为 goes。在交际过程中, 教师用正确的语序: Li Ming goes first.来回答学生, 而且可 以强调或重读 goes 和 first, 让学生觉得 first 应置于句末。 学生交流多了, 就会不知不觉地把不正确的语序改正过来, 无形中习得了正确的语序。教师的这种潜移默化的引导方 法是很受学生欢迎的, 因为在课堂教学中, 学生存在着面 子问题。如果不间断地纠错, 再不在乎面子的学生也会觉 得没面子。所以对于这类有利于交际的纠错方法, 教师要 在教学实践中不断地发掘、积累并发扬光大。

而在非口语交际中, 由于对方不在现场, 交际中所产 生的语言错误无法用弥补的办法来得到纠正, 因此错误往 往会造成误解。例如有这样一则语法错误: I went to the cinema every week.。本来 went 应该是 go, 由于用了 went, 交际对方会误解成是过去的每个星期去看电影, 但自交际 这一刻起到将来就不去或者不一定去看电影。对于此类错 误, 教师绝不能含糊, 应坚决加以纠正。

\section{5. 结语}

在英语教学, 特别是口语教学实践中, 教师对学生的 语言错误应持正确的态度。在口语交际中可以用引导的方 法来帮助学生纠正错误, 要尽量避免直截了当地指出和纠 正错误。在非口语交际中, 教师应当非常严肃认真地对待 和纠正学生的语言错误。 


\section{参考文献(References)}

[1] C. Cao, "Communication culture and basic English teaching," Shangdong Journal of English Teaching, vol. 5, no. 2, pp. 34-37, 1993.

[2] W. Dai, "A comprehensive review of error analysis," Foreign Languages, vol. 11, no. 2, pp. 3-8, 1990.
[3] Z. He and Z. Yan, "Pramatic errors in English communication of Chinese students - pragmatic errors of Chinese and English," Foreign Language Teaching and Research, vol. 8, no. 3, pp. 36-41, 1991.

[4] W. Zhang, An Analysis of the Common Errors by the Chinese in Their Use of English. Beijing: Huaxia Publishing House, 1993. 